\title{
Detection of a large massive circumstellar disk around a high-mass young stellar object in the Carina Nebula ${ }^{\star}$
}

\author{
T. Preibisch ${ }^{1}$, T. Ratzka ${ }^{1}$, T. Gehring ${ }^{1}$, H. Ohlendorf ${ }^{1}$, H. Zinnecker $^{2,3,4}$, R. R. King ${ }^{5}$, \\ M. J. McCaughrean ${ }^{6,5}$, and J. R. Lewis ${ }^{7}$
}

\author{
${ }^{1}$ Universitäts-Sternwarte München, Ludwig-Maximilians-Universität, Scheinerstr. 1, 81679 München, Germany \\ e-mail: preibisch@usm.uni-muenchen.de \\ 2 Astrophysikalisches Institut Potsdam, An der Sternwarte 16, 14482 Potsdam, Germany \\ 3 Deutsches SOFIA Institut, Universität Stuttgart, Pfaffenwaldring 31, 70569 Stuttgart, Germany \\ 4 NASA-Ames Research Center, MS 211-3, Moffett Field, CA 94035, USA \\ 5 Astrophysics Group, College of Engineering, Mathematics and Physical Sciences, University of Exeter, Exeter EX4 4QL, UK \\ ${ }^{6}$ European Space Agency, Research \& Scientific Support Department, ESTEC, Postbus 299, 2200 AG Noordwijk, The Netherlands \\ 7 Cambridge Astronomical Survey Unit, Institute of Astronomy, Madingley Road, Cambridge, CB3 OHA, UK
}

Received 17 January 2011 / Accepted 7 April 2011

\begin{abstract}
Context. The characterization of circumstellar disks around young stellar objects can provide important information about the process of star formation and the possible formation of planetary systems.

Aims. We investigate the spatial structure and the spectral energy distribution of a newly discovered edge-on circumstellar disk around an optically invisible young stellar object that is embedded in a dark cloud in the Carina Nebula.

Methods. The disk object was serendipitously discovered in our deep near-IR imaging survey of the Carina Nebula obtained with HAWK-I at the ESO VLT. Whereas the object was detected as an apparently point-like source in earlier infrared observations, only the superb image quality $\left(F W H M \approx 0.5^{\prime \prime}\right)$ of the HAWK-I data could reveal, for the first time, the peculiar morphology of the object. It consists of a very red point-like central source that is surrounded by a roughly spherical nebula, which is intersected by a remarkable dark lane through the center. We construct the spectral energy distribution of the object from $1 \mu \mathrm{m}$ to $870 \mu \mathrm{m}$ and perform a detailed radiative transfer modeling of the spectral energy distribution and the source morphology.

Results. The observed object morphology in the near-IR images clearly suggests a young stellar object that is embedded in an extended, roughly spherical envelope and surrounded by a large circumstellar disk with a diameter of $\approx 5500$ AU that is seen nearly edge-on. The radiative transfer modeling shows that the central object is highly luminous and thus must be a massive young stellar object, most likely in the range $10-15 M_{\odot}$. The circumstellar disk has a mass of about $2 M_{\odot}$.

Conclusions. The disk object in Carina is one of the most massive young stellar objects for which a circumstellar disk has been detected so far. The size and mass of the disk are very large compared to the corresponding values found for most other similar objects. These results support the assumption that $10-15 M_{\odot}$ stars can form via accretion from a circumstellar disk.
\end{abstract}

Key words. stars: formation - circumstellar matter - stars: pre-main sequence - ISM: individual objects: NGC 3372

\section{Introduction}

During the past dozen years, a substantial number of circumstellar accretion disks around young stellar objects have been spatially resolved with a number of different techniques such as direct optical/infrared imaging, (sub-)mm or radio-mapping, radio-interferometry, and (more recently) infrared interferometry (see, e.g., Stapelfeldt et al. 1998; Zinnecker et al. 1999; Wolf et al. 2003; Leinert et al. 2004; Preibisch et al. 2006; Kraus et al. 2008; Ratzka et al. 2009; Gramajo et al. 2010; Quanz et al. 2010). Spectroscopic observations have also provided important kinematical evidence of accretion disks (see, e.g., Chandler et al. 1995; Bik \& Thi 2004; Wheelwright et al. 2010; Davies et al. 2010). These studies have greatly advanced our understanding of the physics of young stellar objects (YSOs), how the young stars gain their final mass, how the circumstellar matter, in which

* The near-infrared observations for this project were collected with the HAWK-I instrument on the VLT at Paranal Observatory, Chile, under ESO program 60.A-9284(K). planets may be forming, evolves with time and is finally dispersed (see Dullemond et al. 2007).

So far, nearly all well characterized circumstellar disks surround low- or intermediate mass stellar objects, with less than about 5-8 $M_{\odot}$. Only a very limited number of disk detections have been reported around objects with higher masses in the range $M \sim 8-20 M_{\odot}$ (see, e.g., Chini et al. 2004; Jiang et al. 2005; Nielbock et al. 2007; Kraus et al. 2010; Davies et al. 2010). However, in many of these cases the mass estimates for the central star are quite uncertain; the true mass may in fact be considerably smaller than initially estimated (see, e.g., Sako et al. 2005). Another problem is that the observed flattened structure around these more massive young stellar objects are not necessarily accretion disks, from which material is directly transferred to the central star; they may rather represent a thick torus that could be the remnant of a flattened envelope (Allen et al. 2003). Considering even higher stellar masses, $M \gtrsim$ $20 M_{\odot}$, no clear observational evidence for accretion disks has yet been found (see, e.g., Cesaroni et al. 2007). This rarity and lack of convincing disk detections is an important aspect in the 
long-standing discussion about how massive stars form. A fundamental problem is that the strong radiation pressure resulting from the very high luminosity of a massive protostar tends to halt the accretion flow and should thus limit the final stellar mass, at least in the case of spherical accretion (Kahn 1974). More recent theoretical results alleviate this problem by mechanisms such as non-spherical accretion, the "flash-light effect", and "photonbubbles" (see, e.g., Yorke \& Sonnhalter 2002; Krumholz et al. 2009). The more accurate treatment of frequency-dependent radiative feedback by Kuiper et al. (2010) suggests that stars with masses well beyond the upper mass limit of spherical accretion can be formed by accretion. However, due to the limited numerical resolution of these simulations (e.g., 1.27 AU in the study of Kuiper et al. 2010), the reliability of the numerical results is not fully clear. As discussed in Zinnecker \& Yorke (2007), there are still strong indications that the formation of massive stars is not simply a scaled-up version of the low-mass star formation process. Alternative models for the formation of massive stars highlight the importance of the cluster environment during early star formation stages (see, e.g., Bonnell et al. 2004; Krumholz et al. 2010), and processes such as protostellar collisions and mergers may be possible in the central regions of very dense clusters (see, e.g., Bonnell et al. 1998; Bonnell \& Bate 2005; Bally \& Zinnecker 2005; Davies et al. 2006).

In the context of these open questions, every new detection and good characterization of a circumstellar disk around a massive $\left(M \gtrsim 8 M_{\odot}\right)$ young stellar object provides an important new mosaic stone that helps to solve the puzzle of massive star formation. However, observations of massive young stellar objects are strongly hampered by the relative rarity of massive stars, their correspondingly larger average distances (as compared to low-mass stars), and the extremely short timescales on which massive protostars evolve. The expected lifetime of a disk around a massive young stellar object is very small, $\lesssim 10^{5} \mathrm{yrs}$, since the circumstellar material is quickly dispersed by the enormous luminosity and wind power of the young high-mass stars. Furthermore, due to their extreme youth, massive YSOs with disks are still deeply embedded in their natal cloud and thus optically invisible.

The Great Nebula in Carina (NGC 3372, see Smith \& Brooks 2008 , for an overview) is a superb location in which to study the physics of massive star formation. At a distance of $2.3 \mathrm{kpc}$, it represents the nearest southern region with a large population of massive stars, among them several of the most massive $\left(M \sim 100 M_{\odot}\right)$ and luminous stars known in our Galaxy. Most of these very massive stars reside in the clusters Trumpler 14 and 16, which are thought to have ages of a few Myr (Tapia et al. 2003). Recent sensitive infrared, sub-mm, and radio observations showed clearly that the Carina Nebula Complex (CNC) is a site of ongoing star formation. The region contains more than $10^{5} M_{\odot}$ of gas and dust (see Smith \& Brooks 2008; Preibisch et al. 2011a). Several very young stellar objects (Megeath et al. 1996; Mottram et al. 2007) and a spectacular young embedded cluster (the "Treasure Chest Cluster"; see Smith et al. 2005) have been found in the molecular clouds, a deep HST H $\alpha$ imaging survey revealed dozens of jet-driving young stellar objects (Smith et al. 2010a), and Spitzer surveys located numerous embedded protostars throughout the Carina complex (Smith et al. 2010b; Povich et al. 2011). A recent deep wide-field X-ray survey revealed at least $\sim 11000$ young stars in this area (Townsley et al. 2011; Preibisch et al. 2011b). The CNC is thus an ideal target in which to search for very young massive stars.

We have recently used the ESO $8 \mathrm{~m}$ Very Large Telescope to perform the largest and deepest near-infrared survey of the CNC obtained so far. In our images, we discovered an optically invisible infrared source with a very interesting morphology, which seems to be a young stellar object surrounded by a huge circumstellar disk seen nearly edge-on. It will be referred to as the "disk object" in the following text. In this paper we first discuss the morphology of the object and its environment, and then determine its spectral energy distribution from the near-infrared to the sub-mm range. We compare the spectral energy distribution to models of young stellar objects surrounded by circumstellar disks and envelopes. Finally, we present the results of our comprehensive and detailed radiative transfer simulations in which we simultaneously modeled the spectral energy distribution and the observed near-infrared morphology of the object in order to determine basic parameters of the central young stellar object and the physical properties of the surrounding disk and envelope.

\section{Observations}

The near-infrared data presented in this paper were obtained in the context of our large survey of the Carina Nebula (see Preibisch et al. 2011c, for more details) with the instrument HAWK-I (see Kissler-Patig et al. 2008) at the ESO $8 \mathrm{~m}$ Very Large Telescope. HAWK-I is equipped with a mosaic of four Hawaii 2RG $2048 \times 2048$ pixel detectors with a scale of $0.106^{\prime \prime}$ per pixel. All data were processed and calibrated by the Cambridge Astronomical Survey Unit using pipeline software originally designed for the analysis of the UKIRT Infrared Deep Sky Survey. In the final images, objects as faint as $J \sim 23 \mathrm{mag}$, $H \sim 22 \mathrm{mag}$, and $K_{\mathrm{s}} \sim 21 \mathrm{mag}$ are detected at $S / N=3$.

The parts of the HAWK-I mosaic in which the disk object is located were obtained in the night of 27 January 2008. The image quality of these specific frames is particularly good; we measured the PSF size for several point-like sources near the disk object and found $F W H M$ values of $0.37^{\prime \prime}$ in the $K_{\mathrm{s}}$-band, $0.45^{\prime \prime}$ in the $H$-band, and $0.52^{\prime \prime}$ in the $J$-band. This excellent image quality (for ground-based seeing-limited observations) was essential for the detection and morphological characterization of the disk object.

In order to get more comprehensive information about the disk object we also analyzed archival data from the Spitzer Space Telescope (IRAC and MIPS maps) and optical Hubble Space Telescope (HST) images taken with WFPC2 and ACS.

Finally, we also used our own deep sub-mm map of the CNC (Preibisch et al. 2011a) that we recently obtained with the Large APEX Bolometer Camera LABOCA at the APEX telescope. This map traces the $870 \mu \mathrm{m}$ emission at $18^{\prime \prime}$ angular resolution

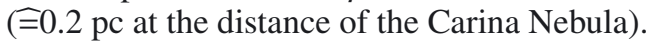

\section{Discovery of the disk object in the dark cloud near Cr 232}

The disk object was discovered in our visual inspection of the HAWK-I images due to its peculiar morphology. It is the brightest member of a group of very red, deeply embedded objects that are surrounded by extended nebulosity, as shown in Fig. 1. The disk object is located at $\mathrm{J} 2000$ coordinates $\alpha=10^{\mathrm{h}} 44^{\mathrm{m}} 31.06^{\mathrm{s}}$, $\delta=-59^{\circ} 33^{\prime} 10.5^{\prime \prime}$ and is seen as an extended, roughly spherical nebulosity with a diameter of about $4^{\prime \prime}$. The nebulosity is intersected by a prominent dark lane that divides it into two similarly large hemispheres. The dark lane can be clearly traced over a distance of $3^{\prime \prime}$, corresponding to about 7000 AU. The global morphology of this object is similar to the famous "Butterfly 
T. Preibisch et al.: A large massive circumstellar disk surrounding a high-mass young stellar object in the Carina Nebula
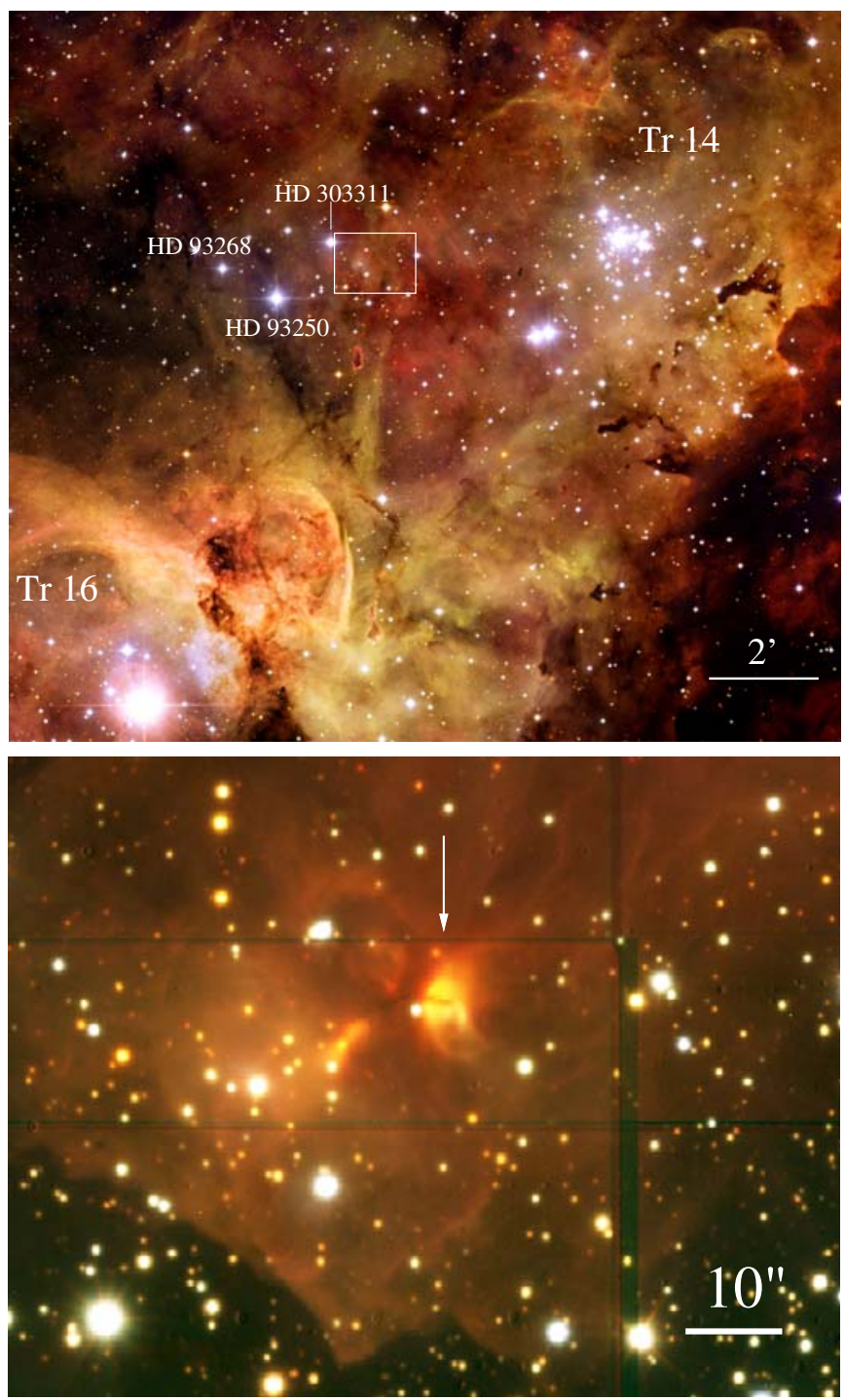

Fig. 1. Upper image: optical image of the central part of the Carina Nebula, reproduced from the ESO photo release 1031 (http://www. eso.org/public/images/etamosaicnm2/). North is up and east to the left. The box shows the area covered by the near-infrared HAWKI image below. Lower image: RGB composite image constructed from the $J$ - (blue), $H$ - (green), and $K_{\mathrm{s}}$-band (red) HAWK-I images of the area around the disk object. (Note: the horizontal and vertical dark streaks are artifacts related to the dither pattern and the mosaicing process.)

Nebula" in Taurus, (a circumstellar disk surrounding a T Tauri star, see Wolf et al. 2003) or the "Silhouette Disk" around a young star in M 17 (Chini et al. 2004), and is discussed in more detail in Sect. 4.

\subsection{The environment of the disk object}

In the optical images of this region (see Fig. 1) the disk object is completely invisible since it is embedded in a dark cloud in the region of the clustering Collinder 232. In order to show the structure of this cloud, we plot in Fig. 2 the contours of the $870 \mu \mathrm{m}$ emission (tracing the cold dust in the cloud) on the $8 \mu \mathrm{m}$ Spitzer image (tracing the surface of the cloud). The south-eastern edge of the cloud is very sharp, straight and bright; its orientation is nearly perpendicular to the direction towards $\eta$ Car. This suggests that this cloud edge is an ionization front caused by the strong UV irradiation from $\eta$ Car and the O-type stars in Tr 16.

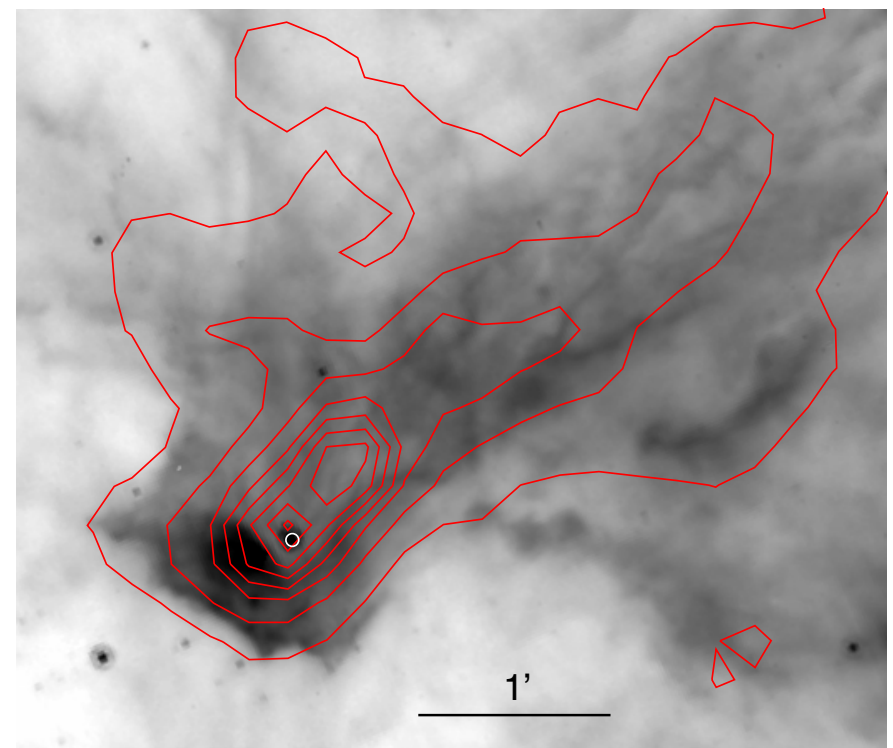

Fig. 2. Negative grayscale representation of the $8 \mu \mathrm{m}$ Spitzer image of the cloud in which the disk object is embedded, with overplotted contours of the $870 \mu \mathrm{m}$ LABOCA map. The field-of-view is $\approx 4.5^{\prime} \times 3.8^{\prime}$; north is up and east to the left. The sub-mm contour levels increase from $0.06 \mathrm{Jy} /$ beam to $1.5 \mathrm{Jy} /$ beam in equal steps. The position of the disk object is marked by the white circle.

The sub-mm emission of this cloud shows two peaks. The southern peak agrees very well with the position of the disk object, suggesting that this object dominates the sub-mm emission of this peak. The second, northern, peak is found 19" east and $23^{\prime \prime}$ north of the disk object. It coincides with the center of the optical dark cloud and a corresponding minimum in the $8 \mu \mathrm{m}$ surface brightness (see Fig. 2). No embedded infrared sources are seen near the center of this second sub-mm peak.

\subsection{Previous detections of the disk object as an unresolved point-like source}

Since the disk object is a relatively bright infrared source, it was detected in many previous infrared observations of this region, but the details of its spatial structure always remained completely unresolved. Only due to the superior image quality of our HAWK-I data that provide an angular resolution of $\sim 0.5^{\prime \prime}$, its peculiar morphology could be resolved.

Figure 3 shows a small part of the $H$-band HAWK-I image around the disk source in which the locations of other sources are marked. The HAWK-I images show a bright point-like source only $2.15^{\prime \prime}$ to the south-east of the disk object. Its PSF shows no indications of being extended and it is clearly separated from the nebulosity surrounding the disk object. We thus believe that this point source is an unrelated star. Based on its position of $\alpha=$ $10^{\mathrm{h}} 44^{\mathrm{m}} 31.3^{\mathrm{s}} \delta=-59^{\circ} 31^{\prime} 11^{\prime \prime}$ we will refer to it as star $31.3-11$ in the following. This star is optically visible and much brighter than the disk object in the $J$-band, but less bright than the disk object in the $K_{\mathrm{s}}$-band. These rather blue colors may suggest it to be a foreground star.

Inspection of the 2MASS images shows that the disk object and this nearby point source appear there as an unresolved blend, listed as 2MASS-J10443122-5933113 in the point source cata$\log$. Its position is just between the disk object and the southeastern point source (as expected). 


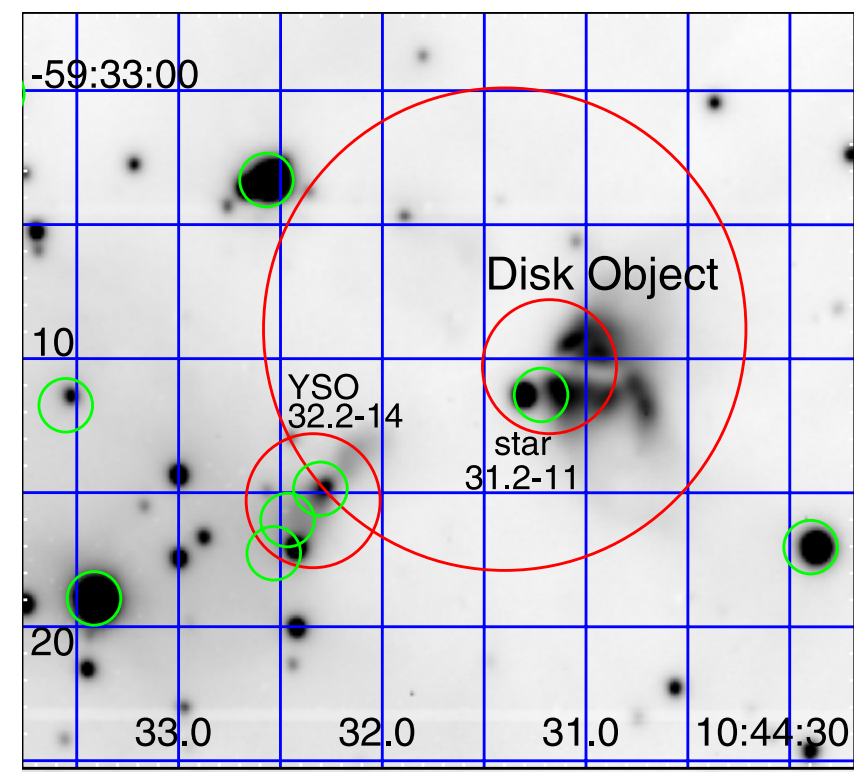

Fig. 3. $H$-band HAWK-I image of the area around the disk object. The small (green) circles with $2^{\prime \prime}$ diameter mark the positions of the objects listed in the 2MASS point source catalog. The $5^{\prime \prime}$ diameter (red) circles mark the locations of the two $10.4 \mu \mathrm{m}$ sources detected by Mottram et al. (2007), and the 18" diameter (red) circle marks the peak of the sub-mm emission as seen in our LABOCA map. A grid of J2000 coordinates is shown.

The disk object is associated with the MSX source G287.4779-00.5463, which appears clearly extended (FWHM $\left.40^{\prime \prime}\right)$ in the MSX images. The object was also detected as a faint diffuse nebulosity in the thermal-infrared study of the Carina nebula by Rathborne et al. (2002), where it is denoted as source "N4". These authors performed a careful analysis of the MSX and IRAS data with the aim to separate the flux from the compact infrared source from the surrounding diffuse emission and derived the spectral energy distribution of the source from $8 \mu \mathrm{m}$ to $100 \mu \mathrm{m}$ (their Fig. 12). They modeled the object with a twocomponent blackbody fit to the SED and derived a total luminosity of $41000 L_{\odot}$ and a spectral type of O9-09.5 for the central object. This result was the first hint that the disk object is actually a massive young stellar object.

Mottram et al. (2007) performed $10.4 \mu \mathrm{m}$ mid-infrared observations of G287.4779-00.5463. Their images were obtained with TIMMI2 at the $3.6 \mathrm{~m}$ ESO telescope and thus provide much better spatial resolution $\left(\sim 1^{\prime \prime}\right)$ than the MSX images. They found two compact mid-infrared sources (these are marked in Fig. 3) associated to G287.4779-00.5463. The brighter of these midinfrared sources coincides perfectly with the disk object, while the fainter one can be identified with another embedded object with surrounding diffuse nebulosity seen in our HAWK-I images about $11^{\prime \prime}$ to the south-east of the disk object. Based on its J2000 coordinates of $\alpha=10^{\mathrm{h}} 44^{\mathrm{m}} 32.3^{\mathrm{s}} \delta=-59^{\circ} 33^{\prime} 14^{\prime \prime}$, we will refer to this second embedded object as YSO 32.2-14 in the following.

The angular separation of the disk object and YSO 32.2-14 is large enough that they are easily discerned in the HAWK-I, 2MASS, Spitzer, and TIMMI2 images. For MSX and IRAS, however, these two objects cannot be separated and thus the fluxes derived for wavelengths beyond $10 \mu \mathrm{m}$ are contaminated by the contribution of the second embedded object.

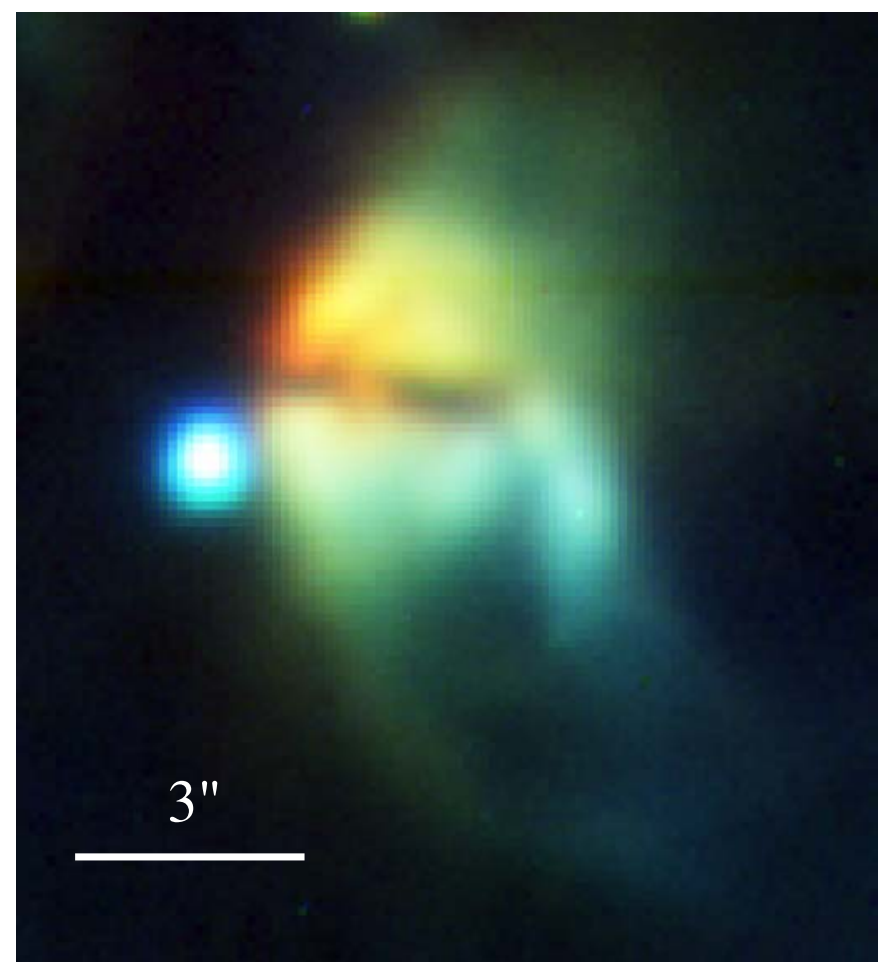

Fig. 4. RGB composite image constructed from the $K_{\mathrm{s}^{-}}, H$-, and $J$-band HAWK-I images.

\section{Morphology and spectral energy distribution of the Disk Object}

\subsection{Near-infrared morphology}

A close-up view of the disk object as seen in our HAWK-I images is shown in Fig. 4. The main morphological structures can be described as follows:

- The general morphology is that of an approximately spherical nebulosity with a diameter of about 4 " (corresponding to $9200 \mathrm{AU}$ ) and a small degree of elongation, approximately along the north-south direction.

- The nebulosity is divided into a northern and a southern lobe (hemisphere) by a prominent dark central lane. The width of the dark lane is $\approx 1.0^{\prime \prime}(2300 \mathrm{AU})$ in the $J$-band image, $\approx 0.5^{\prime \prime}$ (1150 AU) in the $H$-band image, and $\approx 0.3^{\prime \prime}$ (690 AU) in the $K_{\mathrm{s}}$-band image.

- While the central lane appears completely dark in the $J$ band, a faint central object becomes visible in the $H$ - and $K_{\mathrm{S}}$-band.

- While the nebulosity has a rather sharp eastern edge, the western part is much more diffuse. This suggests a windswept morphology from some agent to the east of the object. We note that there are two known massive stars in this direction that may be responsible for this effect: the $\mathrm{O} 5$ star HD 303311, 48" east and 15" north of the disk object (at a projected distance of $0.56 \mathrm{pc}$ ), and the O3 star HD 93250 $105^{\prime \prime}$ east and $44^{\prime \prime}$ south of the disk object (at a projected distance of $1.26 \mathrm{pc}$ ).

- The south western part of the nebulosity shows some kind of "tail" pointing towards the south. This structure breaks the otherwise high degree of symmetry of the disk object. As this "tail" is the only part of the infrared nebulosity that is visible in the optical HST images, it might be related to foreground nebulosity. 
T. Preibisch et al.: A large massive circumstellar disk surrounding a high-mass young stellar object in the Carina Nebula

\subsection{Near-infrared photometry}

Photometric calibration of the HAWK-I images was achieved via bootstrapping from aperture photometry measured in the HAWK-I images for the star 2MASS J10442989-5933170; this star is about $10^{\prime \prime}$ to the south-west of the disk object, appears well isolated, and has 2MASS magnitudes of $J=14.272 \pm 0.04$, $H=13.376 \pm 0.04, K_{\mathrm{s}}=13.015 \pm 0.05$.

For the bright star 31.3-11 near the disk object we determined magnitudes of $J \approx 15.3, H \approx 14.6$, and $K_{\mathrm{s}} \approx 14.7$. The rather bluish color of this star $\left(H-K_{\mathrm{s}}=-0.1\right)$ suggests a very low level of extinction and may imply that this object is located in front of the dark cloud. It also implies that its contribution to the mid- and far-infrared fluxes for the disk object will most likely be very small and can be neglected.

For the disk object, we determined the following measurements: the peak surface brightness in the northern/southern lobes is $17.85 / 16.97 \mathrm{mag} / \mathrm{sqarcsec}$ in the $J$-band, $15.03 /$ $15.01 \mathrm{mag} / \mathrm{sqarcsec}$ in the $H$-band, and $12.44 / 13.13$ $\mathrm{mag} / \mathrm{sqarcsec}$ in the $K_{\mathrm{s}}$-band. The ratio of the integrated fluxes of the northern and southern lobes is $0.62: 1$ in the $J$-band, 0.94:1 in the $H$-band, and $1.47: 1$ in the $K_{\mathrm{s}}$-band. The magnitudes of the central source in the $H$ - and the $K_{\mathrm{s}}$-band (measured in circular apertures with 2.5-pixel radii from which the average of the fluxes measured in two equally large apertures placed just to the east and west of the center in the dust lane was subtracted to account for the local background) are found to be $K_{\mathrm{s}} \sim 16.1, H \sim 19.6, J>21.8$; note that the errors of these measurements are substantial due to the complicated background. If we assume that the observed emission is actually the central young stellar object, the observed color $H-K_{\mathrm{s}} \approx 3.5$ and the assumption that the intrinsic color of the central star should be $\left(H-K_{\mathrm{s}}\right)_{0} \leq 0.1$ lead to an extinction estimate of $A_{\mathrm{V}}>54$ mag. However, we note that the central patch in the images may also just be reflected light from the innermost parts of the circumstellar disk; in that case, the true extinction and optical depth to the central source would be much higher.

\subsection{Morphology at other wavelengths}

In all optical HST images, star 31.3-11 is clearly visible, but the disk object cannot be seen. Only the "tail" at the south-western edge of the disk object is visible in the F555W and F850LP image, suggesting that it may represent reflected light from a foreground cloud that is unrelated to the disk object.

In Fig. 5 we compare the Spitzer IRAC images to our HAWK-I near-infrared images and the HST F555W image. Although the IRAC images do not have enough angular resolution to show the structure of the disk object, it can be seen that the optically invisible disk object strongly brightens with increasing wavelength. The same is true for YSO 32.2-14.

\subsection{Construction of the spectral energy distribution}

We assembled the spectral energy distribution of the disk object from the images and literature data discussed above. For our HAWK-I data we performed aperture photometry using an aperture diameter of about 5", excluding star 31.3-11. For the Spitzer IRAC data an aperture diameter of 7 " was used. Due to the lower angular resolution of the IRAC data, the disk object and star 31.3-11 cannot be separated. However, as the $K_{\mathrm{s}}$-band flux of star $31.3-11$ is only $8.8 \times 10^{-4} \mathrm{Jy}$, i.e. 40 times lower than that of the disk object and since star 31.3-11 shows quite blue colors, we are confident that its contribution to the emission in the IRAC bands can safely be neglected. The second embedded
Table 1. Spectral energy distribution of the disk object.

\begin{tabular}{lcccc}
\hline \hline $\begin{array}{l}\lambda \\
{[\mu \mathrm{m}]}\end{array}$ & $\begin{array}{c}F_{v} \\
{[\mathrm{Jy}]}\end{array}$ & $\begin{array}{c}\text { Aperture } \\
\text { diameter }\end{array}$ & Instrument & Reference \\
\hline 1.26 & $0.0013 \pm 0.0003$ & $5^{\prime \prime}$ & HAWK-I & 1 \\
1.62 & $0.0099 \pm 0.0015$ & $5^{\prime \prime}$ & HAWK-I & 1 \\
2.15 & $0.0351 \pm 0.0045$ & $5^{\prime \prime}$ & HAWK-I & 1 \\
3.6 & $0.113 \pm 0.023$ & $7^{\prime \prime}$ & Spitzer & 1 \\
4.5 & $0.237 \pm 0.047$ & $7^{\prime \prime}$ & Spitzer & 1 \\
5.8 & $0.443 \pm 0.089$ & $7^{\prime \prime}$ & Spitzer & 1 \\
8.0 & $0.760 \pm 0.228$ & $7^{\prime \prime}$ & Spitzer & 1 \\
10.4 & $0.35 \pm 0.01$ & $1.2^{\prime \prime}$ & TIMMI & 2 \\
8.28 & $\approx 5.52$ & & MSX & 3 \\
12.13 & $\approx 10.1$ & & MSX & 3 \\
14.65 & $\approx 7.3$ & & MSX & 3 \\
21.3 & $\approx 28.4$ & & MSX & 3 \\
25 & $\sim 50$ & & IRAS & 3 \\
60 & $\sim 300$ & & IRAS & 3 \\
100 & $\sim 233$ & & IRAS & 3 \\
870 & $\$ 2.5$ & $36^{\prime \prime}$ & LABOCA & 1 \\
\hline
\end{tabular}

References. (1) This work; (2) Mottram et al. (2007); (3) Rathborne et al. (2002).

source YSO 32.2-14 is clearly separated from the disk object in the IRAC images (see Fig. 5) and does not contaminate our photometry.

Although the Spitzer MIPS maps also have sufficient angular resolution to clearly separate the disk object from YSO 32.2-14, both objects are heavily saturated and therefore no quantitative photometric analysis is possible. In order to check the reliability of the mid- to far-infrared fluxes determined by Rathborne et al. (2002) from the MSX data, we analyzed and compared the Spitzer MIPS $24 \mu \mathrm{m}$ image and the MSX E-band $(21.3 \mu \mathrm{m})$ image. The MIPS image shows a bright, roughly circular nebulosity with a diameter of about $50^{\prime \prime}$ with three embedded compact, point-like sources. The brightest of these point-like sources is the disk object, the second brightest is YSO 32.2-14, and the third one is the 2MASS source 10443341-5933189 (the bright object near the lower left corner of Fig. 3). In order to determine the $24 \mu \mathrm{m}$ flux of the nebula, we used an aperture with diameter $54^{\prime \prime}$ and excluded circular regions with diameters of $11^{\prime \prime}$ centered on each of the three point-like sources. Subtracting the large-scale background level estimated from several nearby regions, we found a flux of $\approx 46 \mathrm{Jy}$ for the nebula. In the MSX E-band image, the cloud is also clearly visible, but the point-like sources are not resolved. The total flux, measured again in a 54" diameter aperture, is $\approx 95 \mathrm{Jy}$; this is the sum of the emission from the cloud and the embedded point-like sources. Subtracting the $\approx 46 \mathrm{Jy}$ from the nebula as estimated above from the Spitzer map, leaves a total flux of $\approx 49$ Jy for the sum of the three point-like sources. Since the disk object is clearly brighter in the Spitzer maps than the other two point-like sources, the flux of $28.4 \mathrm{Jy}$ determined by Rathborne et al. (2002) for the dominant pointlike source (i.e. the disk object) is a very reasonable value.

We also performed aperture photometry in our LABOCA sub-mm map. In order to approximately isolate the contribution of the disk object from the surrounding cloud, we measured the flux in an aperture with a radius of $18^{\prime \prime}$ (corresponding to the FWHM of the beam) and subtracted the scaled background flux estimated from the median flux level in an annulus with 1.1 and 1.5 times the aperture radius. This yielded a flux of $2.5 \mathrm{Jy}$. As the rather large aperture partly includes YSO 32.2-14, this value should be regarded as an upper limit to the sub-mm flux from the disk object. 


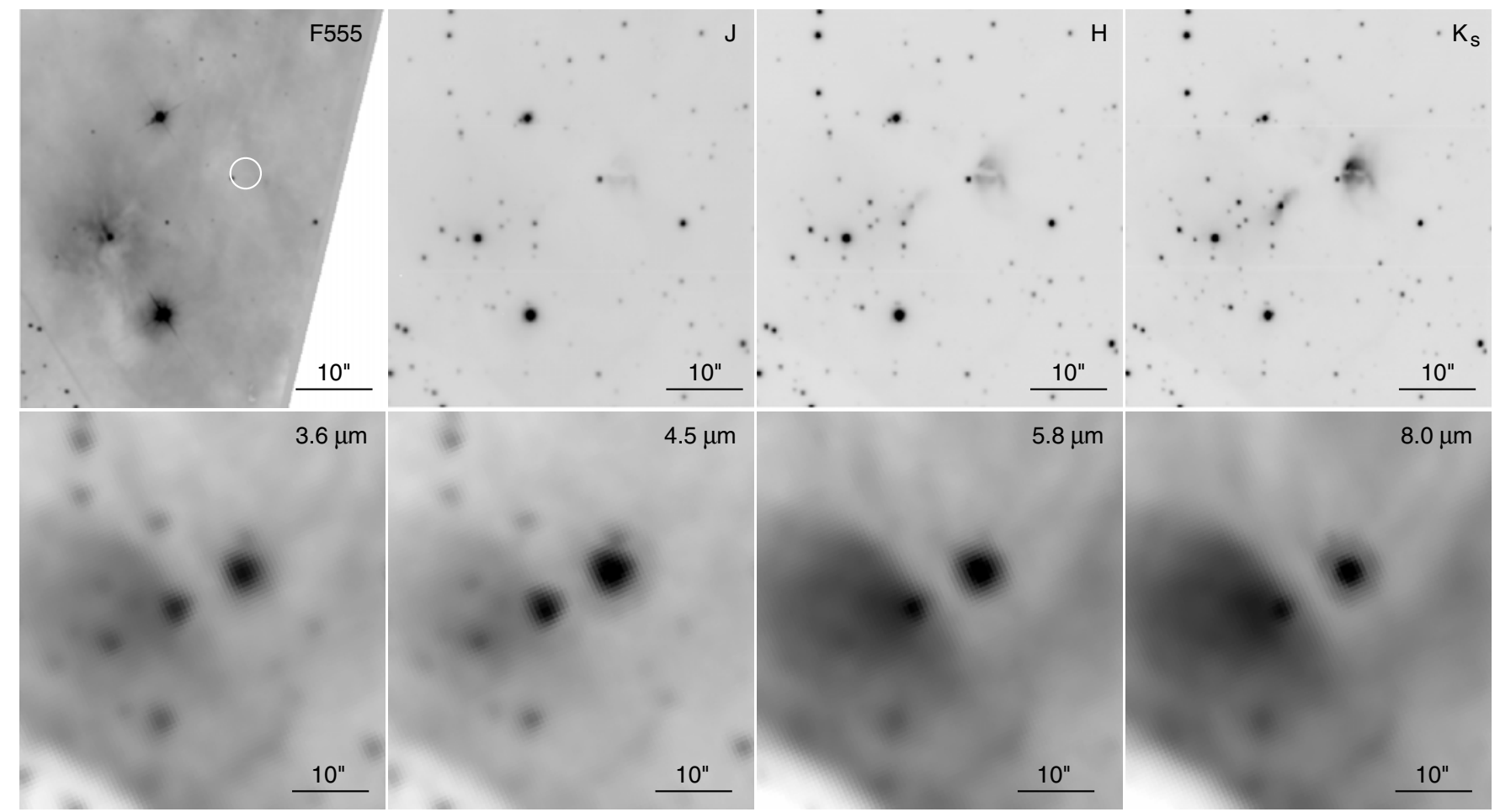

Fig. 5. The disk object and its surroundings seen at different wavelengths. The upper left panel shows the optical HST image taken through the filter F555; the position of the (optically invisible) disk object is marked by the white circle. The next three panels show the HAWK-I images, and the lower row shows the Spitzer IRAC images.

Table 2. Spectral energy distribution of the source YSO 32.2-14.

\begin{tabular}{lcccc}
\hline \hline $\begin{array}{l}\lambda \\
{[\mu \mathrm{m}]}\end{array}$ & $\begin{array}{c}F_{v} \\
{[\mathrm{Jy}]}\end{array}$ & $\begin{array}{c}\text { Aperture } \\
\text { diameter }\end{array}$ & Instrument & Reference \\
\hline 1.26 & $0.00053 \pm 0.00011$ & $2^{\prime \prime}$ & HAWK-I & 1 \\
1.62 & $0.0024 \pm 0.0036$ & $2^{\prime \prime}$ & HAWK-I & 1 \\
2.15 & $0.0089 \pm 0.0013$ & $2^{\prime \prime}$ & HAWK-I & 1 \\
3.6 & $0.053 \pm 0.011$ & $5^{\prime \prime}$ & Spitzer & 1 \\
4.5 & $0.105 \pm 0.021$ & $5^{\prime \prime}$ & Spitzer & 1 \\
5.8 & $0.219 \pm 0.044$ & $5^{\prime \prime}$ & Spitzer & 1 \\
8.0 & $0.390 \pm 0.117$ & $5^{\prime \prime}$ & Spitzer & 1 \\
10.4 & $0.24 \pm 0.01$ & $1.2^{\prime \prime}$ & TIMMI2 & 2 \\
\hline
\end{tabular}

References. (1) This work; (2) Mottram et al. (2007).

The SED of the disk object (see Fig. 6) rises steeply from $1.26 \mu \mathrm{m}$ to $8 \mu \mathrm{m}$. The spectral index measured between $2.15 \mu \mathrm{m}$ and $8 \mu \mathrm{m}$ is $\alpha=0.878$; the object thus qualifies as a "class I source", i.e. a very young object that is embedded in large amounts of circumstellar material.

We note again that the reliability of the fluxes is quite different in the different wavelength ranges. The near- and midinfrared fluxes derived from the HAWK-I and Spitzer data as well as the TIMMI2 flux are highly reliable and not contaminated by other infrared sources. The MSX and IRAS fluxes for the object derived by Rathborne et al. (2002) are less reliable due to the uncertain local background correction and may be contaminated by emission from YSO 32.2-14. The LABOCA flux is also uncertain since it may contain significant amounts of large-scale nebula emission and is therefore regarded as an upper limit in our modeling described below.

A final caveat comes from the fact that the individual points of the SED were not obtained simultaneously. It is well established that many young stellar objects show considerable infrared variability by factors of up to $\sim 2$ or even more, on timescales between days and decades; these variations are thought to be caused by changes in the structure of the inner disks (e.g., Flaherty \& Muzerolle 2010). This implies that flux variations by about a factor of 2 could be present and change the shape of the observed SED.

\section{Radiative transfer modeling}

\subsection{Modeling of the spectral energy distribution with the Robitaille models}

The pre-computed set of SED models provided by Robitaille et al. (2007) are now used as a standard tool to estimate stellar and circumstellar parameters of YSOs from the observed SEDs. We therefore choose to fit the observed spectral energy distribution of the disk source with these models as a first step of our analysis. We note that this kind of analysis ignores the morphological information we have available from the near-infrared images. However, since most current studies of YSO circumstellar structures are based on such SED fits without spatially resolved image information, this modeling can tell us what conclusions would be drawn if the direct spatial morphology information from our HAWK-I images were not available.

Using the SED fluxes as listed in Table 1 and fixing the distance to $2.3 \mathrm{kpc}$, the best fit $\left(\chi^{2} / v=1.89\right)$ is found for a model of a young star surrounded by an envelope with the following parameters: $M_{*}=13.3 M_{\odot}, L_{*}=4190 L_{\odot}, M_{\text {env }}=42 M_{\odot}$, $R_{\mathrm{env}}=5.3 \times 10^{4} \mathrm{AU}$, inclination $=69.5^{\circ}$, and foreground extinction of $A_{\mathrm{V}}=5.59 \mathrm{mag}$.

In the 31 further models with $\chi^{2} / v-\chi^{2} / v$ (best) $<2$, the parameters are in the following ranges: $M_{*}=8.11 \ldots 14.3 M_{\odot}$, $L_{*}=1320 \ldots 4950 L_{\odot}, M_{\mathrm{env}}=4.8 \ldots 363 M_{\odot}$, inclination $=18.2^{\circ} \ldots 87.1^{\circ}$, and foreground extinction of $A_{\mathrm{V}}=$ $1.24 \ldots 20.9$ mag. Nineteen of these models have (in addition 
T. Preibisch et al.: A large massive circumstellar disk surrounding a high-mass young stellar object in the Carina Nebula

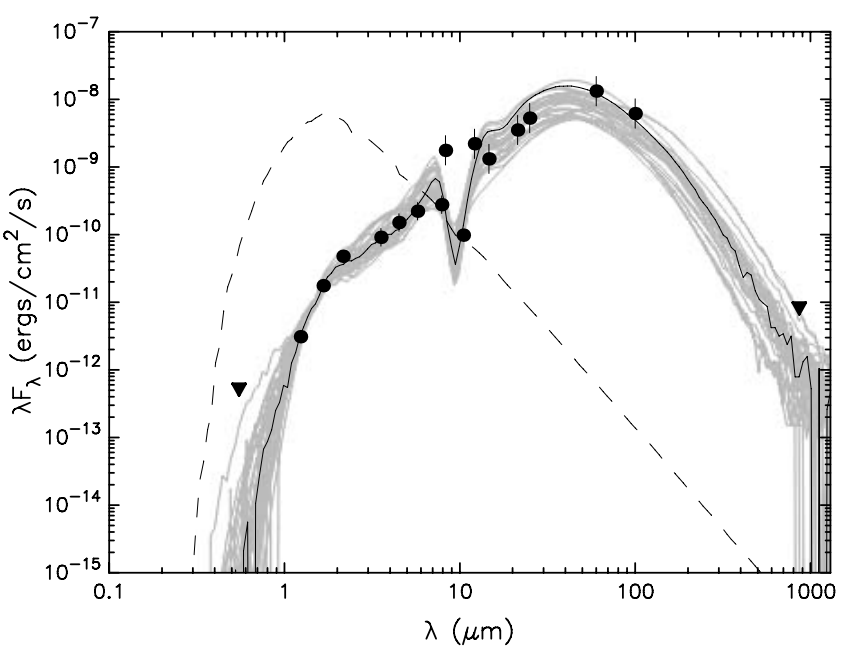

Fig. 6. The 32 best fitting Robitaille SED models compared to the measured fluxes of the disk object. The best fit is shown as a solid line; the dashed line shows the stellar spectrum of the best fit model with the effect of the foreground extinction of $A_{\mathrm{V}}=5.59 \mathrm{mag}$. The grey lines show the other good models as discussed in the text.

to the envelopes) disks with masses $M_{\text {disk }}=8.7 \times 10^{-3} \ldots 4.3 \times$ $10^{-1} M_{\odot}$.

We also performed SED fits excluding the near-infrared fluxes (that are contaminated by scattered light). We found that this produces only very marginal changes of the fitting results. The best model is the same as the second best model in the full SED fit. The parameter ranges for the best 31 models are: $M_{*}=8.28 \ldots 15.6 M_{\odot}, L_{*}=1550 \ldots 6500 L_{\odot}, M_{\text {envelope }}=$ $48.2 \ldots 251 M_{\odot}$, inclination $=18.2^{\circ} \ldots 87.1^{\circ}$, and foreground extinction of $A_{\mathrm{V}}=0 \ldots 35 \mathrm{mag}$.

The rather wide ranges for the stellar and circumstellar parameters demonstrate the large ambiguities of the fits based on the SED only. If we make use of the information deduced from the observed morphology and restrict the selection to models with a nearly edge-on viewing angle $\left(i>75^{\circ}\right)$, we find six different models with the following parameter ranges: $M_{*}=8.45 \ldots 13.2 M_{\odot}, L_{*}=2140 \ldots 4750 L_{\odot}, M_{\text {envelope }}=$ $26 \ldots 93 M_{\odot}, R_{\text {envelope }}=4.4 \times 10^{4} \ldots 1 \times 10^{5}$ AU. Two of these models also have a circumstellar disk; one with a mass of $M_{\text {disk }}=0.027 M_{\odot}$ and radius $R_{\text {disk }}=122 \mathrm{AU}$, and the other one with $M_{\text {disk }}=0.033 M_{\odot}$ and radius $R_{\text {disk }}=8 \mathrm{AU}$.

\section{2. $2 D$ radiative transfer modeling of the SED and morphology}

In the second step of our analysis, we performed a more detailed radiative transfer modeling of the object in which both, the SED and the near-infrared morphology, are properly taken into account. The direct spatial information from the NIR images can resolve many of the ambiguities of the models that are based on SED fits only, and provide a much more physically meaningful model.

We used the 2D radiation transfer code RADMC (Dullemond \& Dominik 2004), which solves the continuum radiative transfer problem in a passive irradiated axisymmetric dusty disk and envelope around a central illuminating star by means of a Monte Carlo algorithm. After the self-consistent computation of the dust temperatures throughout the disk, the SED and images at different wavelengths are computed with a ray-tracing procedure. The density model for the circumstellar disk and envelope we have used in our modeling is defined in cylindrical coordinates $(r, z)$ by the following formulae:

The density of the disk is given by

$\rho_{\text {disk }}=\rho_{\text {disk }, 0}\left(\frac{r}{r_{0}}\right)^{-\alpha} \exp \left[-\frac{z^{2}}{2 h^{2}}\right]$,

where $r_{0}$ is set to the stellar radius and $h$ is the vertical pressure scale height. The parameter $h$ can increase with the radial distance from the center, resulting in a flaring of the disk:

$h(r)=h_{0}\left(\frac{r}{r_{0}}\right)^{\beta}$.

This disk is embedded in a spherical envelope with power-law density gradients:

$\rho_{\text {env }}=\rho_{\text {env }, 0}\left(\frac{r}{R_{\text {env }}}\right)^{\gamma}$ for $r \leq R_{\text {env }}$

and

$\rho_{\mathrm{env}}=\rho_{\mathrm{env}, 0}\left(\frac{r}{R_{\mathrm{env}}}\right)^{\delta}$ for $r>R_{\mathrm{env}}$.

For our computations, we used a grid size of $R=5000 \mathrm{AU}$, 130 radial grid points, 50 angular grid points (with 10 extra angular points to refine the grid near the equator), a gas-to-dust mass ratio of 100:1, and $10^{5}$ photon packets for the Monte Carlo simulations of each model. For the dust opacities we used the updated model of Draine \& Lee (1984) ${ }^{1}$ with $75 \%$ silicate and $25 \%$ graphite. We assumed a canonical grain size distribution $n(a) \propto a^{-3.5}$ with a minimum grain size of $0.005 \mu \mathrm{m}$. For the maximum grain size we used a density dependent value ranging from $a_{\max }=0.25 \mu \mathrm{m}$ for $\rho<10^{-17} \mathrm{~g} / \mathrm{cm}^{2}$ up to $a_{\max }=10 \mu \mathrm{m}$ for $\rho>10^{-13} \mathrm{~g} / \mathrm{cm}^{2}$.

Our density model is described by eleven free parameters: (1) stellar luminosity $L_{*}$; (2) stellar temperature $T_{*}$; (3) disk density power law $\alpha$; (4) disk flaring parameter $\beta$; (5) disk vertical scale height $h_{0} ;(6)$ disk density $\rho_{\text {disk }, 0}$; (7) inner envelope density power law exponent $\gamma$; (8) outer envelope density power law exponent $\delta$; (9) envelope characteristic radius $R_{\text {env }}$; (10) envelope density $\rho_{\text {env }, 0} ;(11)$ inclination $i$. Furthermore, as the object is obviously located inside a dark cloud, we also considered the effects of foreground extinction.

A general problem of such a radiative transfer modeling is that the high dimensionality and the complicated topology of the parameter space make a search for the "best model" very difficult in practice. Simple scanning of the parameter space is not feasible: for example, even an extremely coarse discretization of only 5 different values for each of the 11 parameters would already require the computation (and evaluation) of about 50 million different models.

Another problem is how to evaluate the fit-quality of a specific model. For the comparison of the model SED to the observed SED, a simple $\chi^{2}$ analysis is easy to implement. A quantitative evaluation of the model images, however, is not so straightforward. First attempts to compare the individual pixel values in the model images to those in the observed images were not successful. Instead, we performed a quantitative assessment of the most important morphological features in the images. This was implemented by computing 2D discrete cosine transformations (DCTs) of the model images and comparing 192 DCT coefficients per model image to those derived from the observed

\footnotetext{
1 See

http://www . astro.princeton. edu/ draine/dust/dust.diel. html
} 


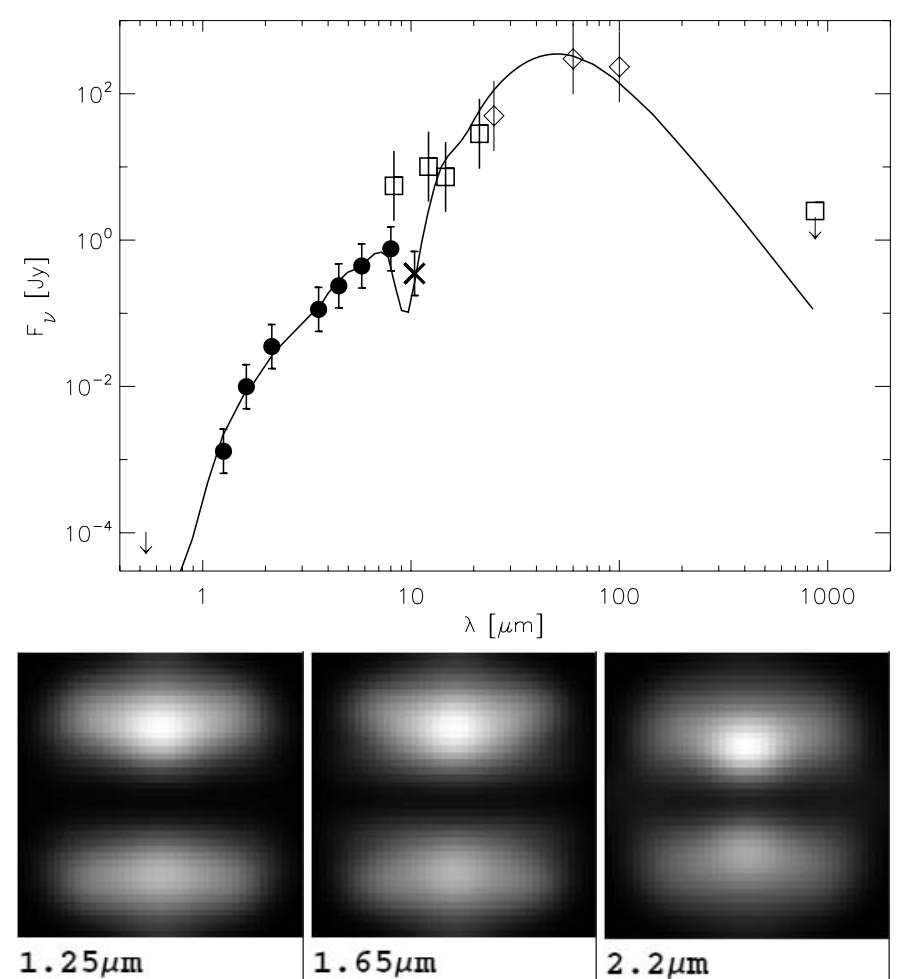

Fig. 7. SED (above) and near-infrared model images (below) computed with RADMC. The solid dots in the SED plot show the HAWK-I and Spitzer data points, the open squares the MSX data, the thick $\mathrm{X}$ symbol the TIMMI2 measurement, the open diamonds the IRAS fluxes, and the open square the LABOCA flux. A square-root intensity scaling is used for the model images.

images. In this way we made sure that model images classified as "good fits" showed a central dark lane and a roundish nebulosity above and below this lane.

In the first part of our modeling, we computed several thousand models to explore the main effects of the individual parameters on the fit quality. Based on these initial results, we then implemented a "genetic algorithm", in which small random changes of some of the parameters of good models are introduced in order to find an even better model. In total, more than 150000 models were computed.

It was very easy to find models that reproduce the SED very well. However, the images of these models deviate considerably from the observed images: they generally produced a much thicker dark lane, and often the shape of the upper and lower reflection nebulosity was more cone-like than hemisphere-like (as observed). On the other hand, we also found several models that reproduced the near-infrared morphology quite well; these models, however, could not reproduce the shape of the observed SED between $2 \mu \mathrm{m}$ and $\sim 20 \mu \mathrm{m}$.

We finally found several models that reproduce both, SED and images, in an acceptable way (although none of these models could simultaneously reproduce the SED and the nearinfrared morphology perfectly). One of these "reasonably good" models is shown in Fig. 7. The model parameters are:

- stellar Luminosity: $L_{*}=12000 L_{\odot}$

- stellar temperature: $T_{*}=22000 \mathrm{~K}$;

- disk density power law: $\alpha=2.25$;

- disk flaring parameter: $\beta=1.25$;

- disk vertical scale height: $h_{0} / r_{0}=0.2$;

- total disk mass: $M_{\text {disk }}=1.78 M_{\odot}$;
- inner envelope density power law exponent: $\gamma=1.3$;

- outer envelope density power law exponent: $\delta=-4.5$;

- envelope characteristic radius: $R_{\mathrm{env}}=1500 \mathrm{AU}$;

- total envelope mass: $M_{\mathrm{env}}=0.16 M_{\odot}$;

- inclination: $i=87^{\circ}$.

The model assumes a foreground (i.e. interstellar cloud) extinction of $A_{\mathrm{V}}=2.5 \mathrm{mag}$. Comparison of the stellar luminosity and temperature to the pre-main sequence models for massive stars from Bernasconi \& Maeder (1996) suggests a stellar mass of $\approx 12 M_{\odot}$. Such an object should evolve towards a ZAMS star of spectral type $\approx \mathrm{B} 1$.

Similarly good models can be found for the following range of the main parameters: $L_{*} \approx 10000 \ldots 15000 L_{\odot}, M_{\text {disk }} \approx$ $1.8 \ldots 4.7 M_{\odot}$, and $M_{\text {env }} \approx 0.16 \ldots 0.5 M_{\odot}$. The range of values for the vertical disk scale height in the good models is $h_{0} / r_{0}=0.1 \ldots 0.2$; this agrees well with the typical scale heights found for circumstellar disks around young stars (see Dullemond $\&$ Dominik 2004) and clearly shows that the spatial configuration of the circumstellar material is that of a geometrically thin disk, and not a thick torus (that would be characterized by $\left.h_{0} / r_{0}>0.5\right)$. The luminosity range suggests that the most likely mass of the central object is in the range $\approx 10 \ldots 15 M_{\odot}$.

One fundamental difference between this model (for SED and near-IR image morphology) and the Robitaille models (for the SED only) is the presence of a massive and optically thick circumstellar disk. Such a disk is not required for a good fit to the SED alone, but its presence is clearly established from the near-infrared images. Another notable difference is seen in the values of the stellar luminosity, which is considerably higher in our RADMC models compared to the Robitaille SED models. This difference is related to the so-called "flashlight effect" first discussed by Yorke \& Bodenheimer (1999). It results from the highly non-isotropic distribution of radiative flux that occurs when a dense, optically thick and flat circumstellar disk is present around an illuminating central source. At shorter wavelengths (for which the disk is optically thick), the radiation field is strongly concentrated toward the polar directions. In such a situation, the total observed outgoing radiation flux is a strong function of the viewing angle; the integrated observed flux for nearly edge-on inclinations $i \approx 90^{\circ}$ can be considerably smaller than the integrated observed flux measured from a more poleon view position (see Yorke \& Sonnhalter 2002). In the case of the Carina disk object, the presence of the dark central lane directly shows that much of the stellar light is removed from our viewing direction and scattered into directions perpendicular to the line-of-sight. Therefore, a high stellar luminosity is required to reproduce the observed flux seen in our edge-on viewing direction. The Robitaille models (that use only the SED as input), on the other hand, have no such optically thick disk around the star and thus assume a much more isotropic radiation field. As a consequence, these models underestimate the luminosity of the object.

It has to be emphasized that the detection and the 2D characterization of the disk object was only possible due to the superb image quality of the HAWK-I data. In order to check how strongly our modeling results depend on the angular resolution, we applied Gaussian filters with different FWHM to the images to see how the observed morphology changes. Degrading the intrinsic resolution of the images $\left(\approx 0.4^{\prime \prime}\right.$ in the $K_{\mathrm{s}}$-band) to a more typical value of $0.7^{\prime \prime}$ is already sufficient to reduce the contrast between the dark lane and the bright lobes to less than $10 \%$. This implies that under "average" seeing conditions the presence of the dark lane (and thus the nature of the object) would no longer 
be clear. Since the dark lane is the strongest observational constraint for our 2D modeling, the existence of a disk would no longer be established.

\section{Summary and conclusions}

Our near-infrared survey of the Carina Nebula led to the serendipitous discovery of an interesting embedded young stellar object that is surrounded by a circumstellar disk and a very large envelope. The presence of the prominent envelope suggests a very early evolutionary stage of the young stellar object. The most notable characteristics of this object derived from our modeling can be summarized as follows:

The central young stellar object has a high estimated mass of about $12 M_{\odot}$; this object is thus one of the very few known cases of massive young stellar objects that are clearly surrounded by a circumstellar disk.

Another outstanding feature is the very large physical size of the disk, which has a radius of $2700 \mathrm{AU}$. This is thus much larger than most disks found around lower-mass young stellar objects (that have typical radii of a at most a few hundred AU).

Finally, our modeling suggests a quite high mass of about $2 M_{\odot}$ for the circumstellar disk, and an additional envelope mass of about $0.2 M_{\odot}$. The total mass of the circumstellar material (within $\$ 10000 \mathrm{AU}$ ) seems to be of the order $\sim 2-3 M_{\odot}$. This is a remarkably high value for the circumstellar material around an individual young stellar object. Also, the ratio between circumstellar mass and stellar mass is high, $\sim 20 \%$. This is much higher than typically found for lower-mass young stellar objects.

Based on these properties, the Carina disk object clearly represents one of the most interesting circumstellar disk detections made so far. Further observations are planned to study this object in more detail. An important point is the uncertainty of the current flux determinations at wavelengths above $\sim 10 \mu \mathrm{m}$, where the angular resolution of the existing data sets does not allow an accurate separation of the disk object from neighboring embedded objects and the surrounding diffuse cloud emission. Although we have tried to account for these effects, it is still possible that the SED fluxes are contaminated by the surrounding nebulosity (as in most other far-infrared observations of embedded objects). Therefore we cannot exclude the possibility that the fluxes from the object are actually somewhat lower, which could lead to smaller values for the disk mass and the mass of the YSO.

The radiative transfer modeling demonstrates the essential value of direct image information. As mentioned above, the detection and characterization of the disk was only possible due to the very good angular resolution of our HAWK-I images and would not have been feasible under more typical seeing conditions. This emphasizes (once again) the importance of angular resolution in the characterization of circumstellar matter around young stellar objects. Images with even higher angular resolution (e.g., by adaptive optics observations) would provide very interesting new information about the width and the shape of the disk shadow. In particular, the chromaticity of the brightness distribution along cuts perpendicular to the disk plane could provide crucial new constraints for a more detailed radiative transfer modeling, and yield new insight into the physical parameters and dust properties of the circumstellar material around this massive protostar.

Acknowledgements. We would like to thank the referee for comments that helped to improve the paper. We gratefully acknowledge funding of this work by the German Deutsche Forschungsgemeinschaft, DFG project number
PR 569/9-1. Additional support came from funds from the Munich Cluster of Excellence: "Origin and Structure of the Universe". This publication makes use of data products from the Two Micron All Sky Survey, which is a joint project of the University of Massachusetts and the Infrared Processing and Analysis Center/California Institute of Technology, funded by the National Aeronautics and Space Administration and the National Science Foundation. This work makes use of observations made with the Spitzer Space Telescope, which is operated by the Jet Propulsion Laboratory, California Institute of Technology under a contract with NASA.

\section{References}

Allen, A., Li, Z.-Y., \& Shu, F. H. 2003, ApJ, 599, 363

Bally, J., \& Zinnecker, H. 2005, AJ, 129, 2281

Bernasconi, P. A., \& Maeder, A. 1996, A\&A, 307, 829

Bik, A., \& Thi, W. F. 2004, A\&A, 427, L13

Bonnell, I. A., \& Bate, M. R. 2005, MNRAS, 362, 915

Bonnell, I. A., Bate, M. R., \& Zinnecker, H. 1998, MNRAS, 298, 93

Bonnell, I. A., Vine, S. G., \& Bate, M. R. 2004, MNRAS, 349, 735

Cesaroni, R., Galli, D., Lodato, G., Walmsley, C. M., \& Zhang, Q. 2007, Protostars and Planets V, 197

Chandler, C. J., Carlstrom, J. E., \& Scoville, N. Z. 1995, ApJ, 446, 793

Chini, R., Hoffmeister, V., Kimeswenger, S. et al. 2004, Nature, 429, 155

Davies, M. B., Bate, M. R., Bonnell, I. A., Bailey, V. C., \& Tout, C. A. 2006, MNRAS, 370, 2038

Davies, B., Lumsden, S. L., Hoare, M. G., Oudmaijer, R. D., \& de Wit, W.-J. 2010, MNRAS, 402, 1504

Draine, B. T., \& Lee, H. M. 1984, ApJ, 285, 89

Dullemond, C. P., \& Dominik, C. 2004, A\&A, 417, 159

Dullemond, C. P., Hollenbach, D., Kamp, I., \& D'Alessio, P. 2007, Protostars and Planets V, 555

Flaherty, K. M., \& Muzerolle, J. 2010, ApJ, 719, 1733

Gramajo, L. V., Whitney, B. A., Gómez, M., \& Robitaille, T. P. 2010, AJ, 139, 2504

Jiang, Z., Tamura, M., Fukagawa, M., et al. 2005, Nature, 437, 112

Kahn, F. D. 1974, A\&A, 37, 149

Kissler-Patig, M., Pirard, J. F., Casali, M., et al. 2008, A\&A, 491, 941

Kraus, S., Preibisch, T., \& Ohnaka, K. 2008, ApJ, 676, 490

Kraus, S., Hofmann, K.-H., Menten, K. M., et al. 2010, Nature, 466, 339

Krumholz, M. R., Klein, R. I., McKee, C. F., Offner, S. S. R., \& Cunningham, A. J. 2009, Science, 323, 754

Krumholz, M. R., Cunningham, A. J., Klein, R. I., \& McKee, C. F. 2010, ApJ, 713, 1120

Kuiper, R., Klahr, H., Beuther, H., \& Henning, T. 2010, ApJ, 722, 1556

Leinert, C., van Boekel, R., Waters, L. B. F. M., et al. 2004, A\&A, 423, 537

Megeath, S. T., Cox, P., Bronfman, L., \& Roelfsema, P. R. 1996, A\&A, 305, 296

Mottram, J. C., Hoare, M. G., Lumsden, S. L., et al. 2007, A\&A, 476, 1019

Nielbock, M., Chini, R., Hoffmeister, V. H., et al. 2007, ApJ, 656, L81

Povich, M. S., Smith, N., Majewski, S. R., et al. 2011, ApJS, in press

Preibisch, Th., Kraus, S., Driebe, T., van Boekel, R., \& Weigelt, G. 2006, A\&A, 458, 235

Preibisch, Th., Schuller, F., Ohlendorf, H., et al. 2011a, A\&A, 525, A92

Preibisch, Th., Hodgkin, S., Irwin, M., et al. 2011b, ApJS, 194, 10

Preibisch, Th., Ratzka, Th., Kuderna, B., et al. 2011c, A\&A, 530, A34

Quanz, S. P., Beuther, H., Steinacker, J., et al. 2010, ApJ, 717, 693

Rathborne, J. M., Burton, M. G., Brooks, K. J., et al. 2002, MNRAS, 331, 85

Ratzka, T., Schegerer, A. A., Leinert, Ch., et al. 2009, A\&A, 502, 623

Robitaille, T. P., Whitney, B. A., Indebetouw, R., \& Wood, K. 2007, ApJS, 169 328

Sako, S., Yamashita, T., Kataza, H., et al. 2005, Nature, 434, 995

Smith, N., \& Brooks, K. J. 2008, Handbook of Star Forming Regions, Vol. II:

The Southern Sky, ASP Monograph Publications, ed. B. Reipurth, 5, 138

Smith, N., Stassun, K. G., \& Bally, J. 2005, AJ, 129, 888

Smith, N., Bally, J., \& Walborn, N. R. 2010a, MNRAS, 405, 1153

Smith, N., Povich, N. S., Whitney, B. A., et al. 2010b, MNRAS, 406, 952

Stapelfeldt, K. R., Krist, J. E., Menard, F., et al. 1998, ApJ, 502, L65

Tapia, M., Roth, M., Vazques, R. A., \& Feinstein, A. 2003, MNRAS, 339, 444

Townsley, L., Broos, P. S., Corcoran, M. F., et al. 2011, ApJS, 194, 1

Wheelwright, H. E., Oudmaijer, R. D., de Wit, W. J., et al. 2010, MNRAS, 408 , 1840

Wolf, S., Padgett, D. L., \& Stapelfeldt, K. R. 2003, ApJ, 588, 373

Yorke, H. W., \& Bodenheimer, P. 1999, ApJ, 525, 330

Yorke, H. W., \& Sonnhalter, C. 2002, ApJ, 569, 846

Zinnecker, H., \& Yorke, H. W. 2007, ARA\&A, 45, 481

Zinnecker, H., Krabbe, A., McCaughrean, M. J., et al. 1999, A\&A, 352, L73 